\title{
ESTIMATION OF THE RATE OF RELEASE OF ADRENAL 17-HYDROXYCORTI- COSTEROIDS IN THE HUMAN BEING BY THE VENOUS CATHETER TECHNIQUE WITH A METHOD FOR DETERMINING PLASMA 17-HYDROXYCORTICOSTEROIDS ${ }^{1}$
}

\author{
By PHILIP K. BONDY 2 AND JOAN R. ALTROCK \\ (From the Departments of Medicine, Emory U. School of Medicine and Grady Memorial Hos- \\ pital, and the Department of Internal Medicine, Yale U. School \\ of Medicine, New Haven, Conn.)
}

(Submitted for publication February 4, 1953; accepted April 17, 1953)

The importance of the role of the adrenal cortex in protein and carbohydrate metabolism and in adjustments to injury has been well established in experimental animals. Studies of the activity of the adrenal cortex in similar situations in human beings have been handicapped by lack of a direct objective method for estimating the rate of secretion of adrenal cortical hormones in the intact man. Since it seemed possible that the intravenous catheter technique might be applicable to this problem, attempts were made to estimate the rate of secretion of the adrenal hormones directly by obtaining blood from the left renal vein, proximal to the entrance of the adrenal vein, and multiplying the arterio-venous difference in concentration of cortical hormones by the renal plasma flow. The method has serious limitations, and, as will be discussed below, is probably not sufficiently accurate to be useful as an experimental clinical tool.

\section{ANALYTICAL METHODS}

At the time that this project was initiated, no specific method was available for the estimation of adrenal cortical steroids in plasma. Attempts were therefore made to use bioassay methods on whole plasma, since it was hoped that this would afford a high degree of specificity, without any danger of loss of hormone during the extractions and manipulations incidental to the use of a chemical assay. The concentration of liver-glycogen-depositing material in human arterial plasma proved to be too small to be detected. For the purposes of the assay, $70 \mathrm{mg}$. of glucose were dissolved in $1.26 \mathrm{ml}$. of plasma $+0.14 \mathrm{ml}$. of ethyl alcohol. This solution was injected subcutaneously in seven $0.2 \mathrm{ml}$. portions into each adrenalectomized mouse according to the schedule of Venning, Kasmin, and

1 Supported by grants from the Division of Metabolism and Endocrinology of the National Institutes of Health, U. S. Public Health Service.

2 Present address: Department of Internal Medicine, Yale U. School of Medicine, New Haven, Conn.
Bell (1). Eight to 10 mice were used for assaying each preparation. In all of the assays, the activity of the plasma was less than that of $0.1 \mathrm{ml}$. of Upjohn aqueous adrenal cortical extract ${ }^{3}$ (equivalent to $20 \mu \mathrm{gm}$. of hydrocortisone). No significant difference could be found between the activity of plasma and saline. As a result of our unsuccessful attempts to apply this type of bioassay to the estimation of the plasma corticosteroid concentration, we have concluded that the quantity of these substances present in plasma is too small to be detected by the Venning technique. This finding is not in agreement with the observation of Paschkis, Cantarow, Walkling, and Boyle who were able to use this method for the measurement of adrenal corticoids in dog plasma (2).

Since bioassay was unsatisfactory, a method was devised for the purification of plasma extracts so that the phenylhydrazine colorimetric reaction of Porter and Silber could be applied (3). The method finally developed is shown in the flow sheet (Table I) and is described in detail below :

Plasma proteins are precipitated, and the lipids simultaneously extracted by adding 15 to $20 \mathrm{ml}$. of plasma to 20 times its volume of a 3:1 mixture of methanol and ether. The plasma is dropped slowly from a pipette into $200 \mathrm{ml}$. centrifuge bottles containing the methanol-ether, agitated by a magnetic stirrer. The stirrer is allowed to run two or three minutes after the precipitation is complete to insure complete extraction. The sample is then centrifuged for 15 minutes at moderate speed; the supernatant, which contains the plasma lipids as well as most of the water-soluble components, is transferred to a $2000 \mathrm{ml}$. Erlenmeyer flask, where it is evaporated to dryness under a stream of air at not more than $55^{\circ} \mathrm{C}$. Evaporation to dryness must be thorough but not unduly prolonged. Hydrocortisone is not destroyed by exposure to large volumes of warm air provided that the $\mathrm{pH}$ is approximately neutral.

Several spatulas of granular tin metal are placed in the two-liter flask, in a separatory funnel, and in each of two heavy duty $40-\mathrm{ml}$. centrifuge tubes. The residue of the plasma extract is dissolved in two successive $10 \mathrm{ml}$. portions of 10 per cent isopropyl alcohol at $\mathrm{pH} 1$ $\left(\mathrm{H}_{2} \mathrm{SO}_{4}\right)$ which are pooled in the separatory funnel, where they are extracted with two $10 \mathrm{ml}$. portions of

\footnotetext{
${ }^{3}$ Kindly supplied by Dr. Dwight Ingle, of the Upjohn Company, Kalamazoo, Mich.
} 


\section{TABLE I}

Schematic flow diagram of method of purifying plasma extracts preparatory to applying the Porler-Silber reaction
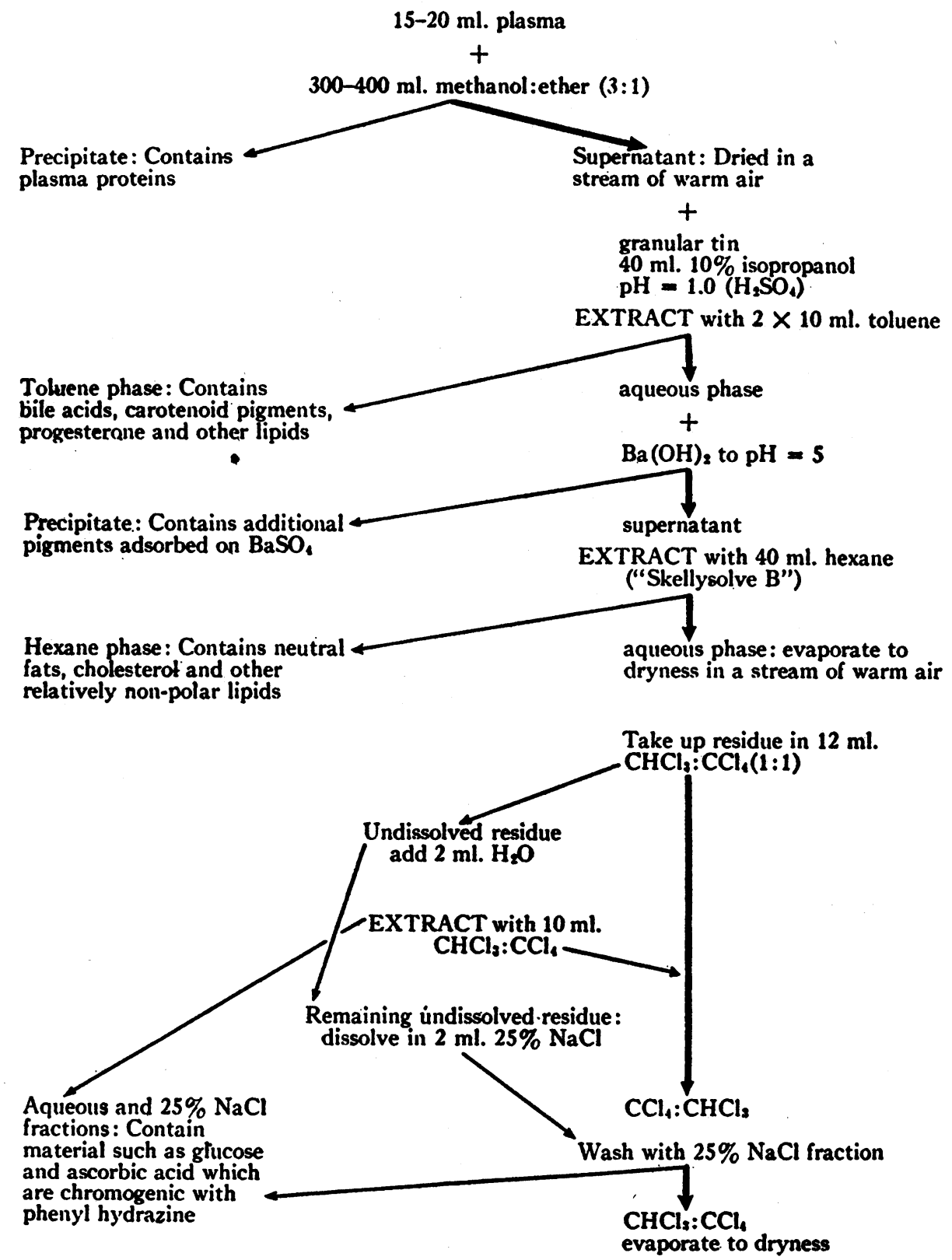

Take up in methanol for colorimetry

toluene. It is inadvisable to shake this mixture too violently, because stable emulsions may form. The toluene-isopropyl alcohol mixture is transferred into the centrifuge tubes. The residue of the plasma extract remaining in the bottom of the two-liter flask is washed twice more with $10 \mathrm{ml}$. portions of 10 per cent isopropyl alcohol, which are added to the centrifuge tubes. The sam- ple is centrifuged at 3000 r.p.m. for 15 minutes, after which the bottom alcohol layer is removed to other centrifuge tubes with a $30 \mathrm{ml}$. syringe equipped with a 4 inch No. 18 needle.

In this extraction, the acid $\mathrm{pH}$ makes bile acids relatively more soluble in the lipid phase than would be the case at neutral $\mathrm{pH}$, and they are transferred to the tolu- 
ene. The same effect probably also occurs with the phenolic steroids such as the estrogens. The toluene phase becomes brilliantly colored with yellow pigments, presumably carotenoids.

It was found that only about 50 per cent of the hydrocortisone could be recovered when the isopropanol: toluene partition was carried out at pH 1. The lost steroid could not be found in either phase, and was, therefore, presumably destroyed in the acid medium. The destructive effect of the low $\mathrm{pH}$ could be completely prevented by the addition of granular tin, as described above.

Using pHydrion paper, the $\mathrm{pH}$ is adjusted to 5 to 6 with a saturated solution of barium hydroxide (about 2 ml.). The resulting precipitate of barium sulfate, with small amounts of adsorbed lipids, is removed by centrifugation at moderate speed for $\mathbf{1 5}$ minutes. The supernatant is shaken with an equal volume of hexane ("Skellysolve B") to complete the removal of non-polar lipids. The agitation should not be too vigorous, since excessive shaking may produce a stable emulsion. The aqueous phase, which contains the corticosteroids and watersoluble materials, is evaporated to dryness in a $125 \mathrm{ml}$. Erlenmeyer flask under a stream of air at a temperature of $55^{\circ} \mathrm{C}$., with precautions to assure adequate, but not prolonged, drying.

It was found that simple extraction of the dry residue with $\mathrm{CCl}_{4}$ failed to dissolve all of the hydrocortisone, probably because of mechanical trapping of the steroid. It was therefore necessary to dissolve the lipid-insoluble material in water in order to permit adequate extraction with the lipid solvent. This is accomplished as follows:

The flask is rinsed with four $3 \mathrm{ml}$. portions of $\mathrm{CCl}_{4}$, which are pooled in the colorimetry tube. The remaining residue is taken up in $2 \mathrm{ml}$. of distilled water and shaken against $10 \mathrm{ml}$. of equal parts of chloroform and carbon tetrachloride. The evaporation flask is rinsed with a $2 \mathrm{ml}$. portion of 25 per cent $\mathrm{NaCl}$ which is put into another separatory funnel and used to back-extract the above $\mathrm{CHCl}_{5}-\mathrm{CCl}_{4}$. All the $\mathrm{CCl}_{4}$ and $\mathrm{CHCl}_{3}-\mathrm{CCl}_{4}$ fractions are pooled in the colorimetry tube and evaporated to dryness, observing the above mentioned precautions. During all manipulations, the sample must be carefully protected against contact with rubber. All solvents are redistilled shortly before using. The air stream used for blowing off the solvents is filtered through a double train of absorbent cotton.

Colorimetry is carried out by adding $0.8 \mathrm{ml}$. of absolute methanol and $1.2 \mathrm{ml}$. of the acid-hydrazine reagent (1.6 mg. of recrystallized phenyl hydrazine per ml. of $20 \mathrm{~N}$ $\mathrm{H}_{2} \mathrm{SO}_{4}$ ). The tubes are cooled as the reagent is added to prevent heating when the methanol and sulfuric acid are mixed. Readings were made using a Coleman Jr. spectrophotometer with an $18 \mathrm{~mm} . \times 125 \mathrm{~mm}$. tube holder modified to reduce the path of light to a slit $8 \mathrm{~mm}$. wide, with an $18 \mathrm{~mm}$. light path. Optical densities are read at 400 and $600 \mathrm{~m} \mu$ exactly five minutes after the addition of the reagents. The sample is then incubated for one hour at $60^{\circ} \mathrm{C}$., cooled rapidly, and re-read at $400 \mathrm{~m} \mu$ and $600 \mathrm{~mm}$. The time required for the color to reach a maximum depends on the temperature at which the reaction proceeds. In the original method of Porter and Silber, the color development was carried out at $80^{\circ} \mathrm{C}$. for 20 minutes. We found it more convenient to develop the color for 60 minutes at $60^{\circ} \mathrm{C}$.

Calculations: The reading at $400 \mathrm{~m} \mu$ before heating corrects for nonspecific color produced by the sulfuric acid in the reagent; and the readings at $600 \mathrm{~m} \mu$ correct for minimal turbidity which may occasionally occur. The corrected optical density is calculated as follows:

$$
O D_{c}=O D_{400}-\left(O D^{1}{ }_{400}+O D_{000}-O D^{1}{ }^{200}\right)
$$

where

$$
\begin{aligned}
\mathrm{OD}_{\mathrm{c}}= & \text { corrected optical density, } \\
\mathrm{OD}_{400}= & \text { optical density observed at } 400 \mathrm{~m} \mu \text { after } 60 \\
& \text { minutes heating, } \\
\mathrm{OD}^{2}{ }_{400}= & \text { optical density observed at } 400 \mathrm{~m} \mu \text { before heating, } \\
\mathrm{OD}_{000}= & \text { optical density at } 600 \mathrm{~m} \mu \text { after } 60 \text { minutes heating, } \\
\mathrm{OD}^{1}{ }^{100}= & \text { optical density at } 600 \mathrm{~m} \mu \text { before heating. }
\end{aligned}
$$

The resulting value of $O D_{c}$ is read from a standard curve for Compound F.4 All solutions are read against a methanol-phenyl hydrazine blank.

The calculation of $O D_{c}$ by this method fails to take into account nonspecific color which may develop during the incubation of sulfuric acid with the plasma extract. The Porter-Silber method may be modified by splitting the reaction into two steps. The colorless phenylhydrazone can first be formed at $\mathrm{pH}=1.0$, and, as a second step, the concentration of $\mathrm{H}_{2} \mathrm{SO}_{4}$ can be brought to $12 \mathrm{~N}$ and the color read immediately. When this is done, the extract need not be incubated at elevated temperatures with high concentrations of $\mathrm{H}_{2} \mathrm{SO}_{4}$, and the nonspecific color formed by sulfuric acid appears at once if it is to appear at all. Since the intensity of the color formed by the one-stage reaction was identical with that produced by the two-stage methods, it was concluded that no increased nonspecific color appears as a result of exposing the plasma to hot $\mathrm{H}_{2} \mathrm{SO}_{4}$, and no correction was included for this possible source of error.

By this method, it is possible to estimate the plasma concentration of neutral lipids giving a color with a peak absorption at $400 \mathrm{~m} \mu$ with acid phenyl hydrazine (Figure 1). Since the reaction is quite specific for steroids with a dihydroxyacetone group on the 17-carbon, the color may be considered as a result of the presence of 17-hydroxycorticosterone (Compound F), 17-hydroxy-11-dehydrocorticosterone (Compound $\mathrm{E}$ or cortisone) and 17-hydroxy-11-desoxycorticosterone (Compound S). Of these, only Compound $\mathrm{F}$ is believed to be present in significant quantities in normal human plasma (4). The extraction procedure completely removes progesterone (which causes a small amount of color at $410 \mathrm{~m} \mu$ ), and all of the watersoluble materials such as ascorbic acid which are strongly chromogenic in the Porter-Silber method. The method will not detect adrenal cortical steroids which have no 17-hydroxyl, and therefore it will not measure corticosterone, which may represent an appreciable fraction of

4 Generously supplied by the Merck Company. 


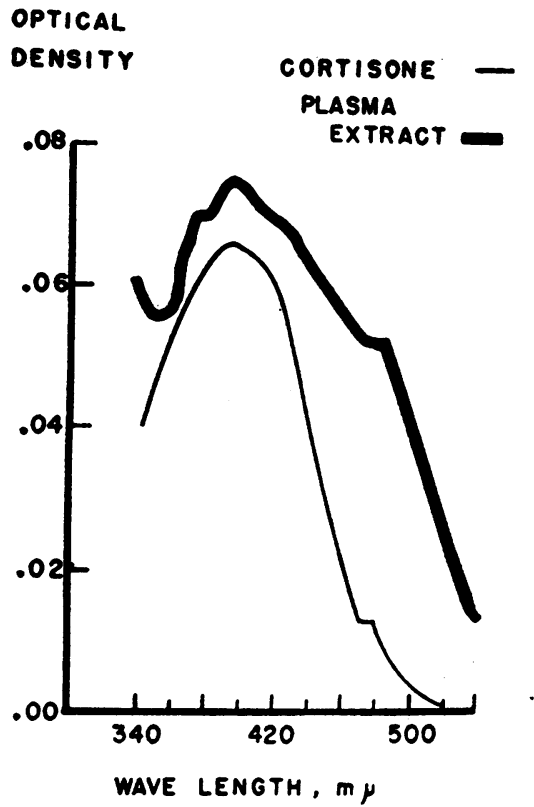

Fig. 1. Absorption Curves of the Phenyl-hydrazone of Plasma Extract Compared with That of $3 \mu \mathrm{Gm}$. of Crystalline Cortisone

the steroid secretion of the human adrenal gland. Since, however, it is estimated that Compound $F$ is the chief adrenal steroid present in the plasma of experimental animals (5) and since Compound $F$ is more potent than corticosterone in promoting glycogen deposition (6) in protecting against adrenalectomy (7), and in maintaining muscular strength (8), it was felt that measurement of Compound $F$ would give an estimate of a large part of the adrenal steroid activity in the plasma.

This method yields recoveries of 85 to 112 per cent of adrenal cortex extract added to plasma, and 60 to 70 per cent of hydrocortisone acetate is recovered after addition to plasma. The standard error of the difference of duplicate analyses equals 5.1 per cent, indicating that a difference of 15.5 per cent between means can be detected with $\mathrm{p}=.05$.

Evidence that the Material Measured is Related to Adrenal Cortical Function. Although it seemed theoretically probable that the chromogen measured by the method described is in fact an adrenal steroid, it was desirable to demonstrate that the material being studied actually originates in the adrenal gland. For this purpose three types of evidence were sought.

In human beings with adrenal cortical insufficiency, the concentration of the chromogen is lower than in normal human beings ( Table II). The fact that it is not entirely absent may mean that the analytical method is not totally specific, and that small amounts of nonspecific (i.e., nonadrenal) chromogenic material are included in the assay. It is also possible that, in some patients with clinical adrenal insufficiency, small amounts of adrenal cortical hormones may still be secreted and appear in minute amounts in the blood. After severe, acute trauma, the chromogenic material is found in the plasma in increased concentration. The range of normal values ( 3 to $13 \mu \mathrm{gm}$. per $100 \mathrm{ml}$.) is similar to that found by Nelson, Samuels, Willardson, and Tyler (4) using another method of preparing plasma for application of the Porter-Silber reaction.

After the administration of adrenocorticotrophic hormone (ACTH), the concentration of the chromogen is increased (Table III). The peak concentration of the chromogen may not have been observed, since Nelson, Samuels, Willardson, and Tyler have shown (4), using

TABLE II

Concentration of 17-hydroxycorticosteroids in the plasma of normal subjects, of patients with adrenal cortical hypofunction, and of patients after severe acute trauma

$\begin{array}{cc} & \text { Normal subjects } \\ \text { Number } & \begin{array}{c}\text { Range } \\ \mu \mathrm{g} . / 100 \mathrm{ml} .\end{array} \\ 12 & 3.0-5.9 \\ 7 & 6.0-8.9 \\ 6 & 9.0-11.9 \\ 5 & 12.0-13.7 \\ \frac{3}{30} & \frac{7.8 \pm 3.3}{\text { (Mean } \pm \text { St. Dev.) }}\end{array}$

Hypoadrenal subjects

Disease

Hypopituitarism*

Addison's Disease*

Addison's Disease*

Adrenalectomy Concentration of
17-OH-corticosteroids Mg. $/ 100 \mathrm{ml}$.

Patients After Severe Acute Trauma

Severe gastrointestinal upset with dehydration and hyponatremia

Fracture, left femur

Lobar pneumonia

Bleeding peptic ulcer

2.0

1.2

3.2

2.3

Acute pyelonephritis

Myocardial infarct

Brachial plexus injury

16.7

16.3

26.3

17.7

26.7

16.3

I.V. Typhoid bacilli for pyrogen therapy 24.3

* These sera were kindly donated by Dr. Gilbert Gordan, University of California Medical School.

a different method of analysis of the plasma, that the peak elevation of the material measured by their method occurs somewhat earlier than the four-hour time interval used in this study.

An additional important point is the fact that the concentration of the chromogen is higher in adrenal vein blood than in peripheral blood. Adrenal vein blood was obtained by anesthetizing rabbits with pentobarbital intravenously, and tying off in succession the renal pedicles, the vena cava below the kidneys, and the vena cava above the adrenal glands. The segment of inferior vena cava thus isolated received drainage from the adrenal glands and also from several small parietal veins. Blood obtained from this segment does not, therefore, represent pure adrenal vein blood, but a large part of the blood so 
obtained does flow from the adrenal glands. The concentration of the chromogen in plasma from a peripheral vein was $4.0 \mu \mathrm{gm}$. per $100 \mathrm{ml}$., whereas that in the isolated inferior vena cava segment was $16.0 \mu \mathrm{gm}$. per 100 $\mathrm{ml}$. in one experiment. In a second experiment, the arterial concentration was $27.6 \mu \mathrm{gm}$. per $100 \mathrm{ml}$., whereas adrenal vein plasma contained $188 \mu \mathrm{gm}$. per $100 \mathrm{ml}$.

In view of this evidence, it seems probable that most of the material measured by the method described originates in the adrenal gland, and consists, in fact, of one or more of the 17-hydroxycorticosteroids.

Experimental Design. The catheter was directed, under fluoroscopic control, through the left brachial vein, via the superior vena cava and right atrium, into the inferior vena cava, and thence into the left renal vein. It was necessary to obtain blood from the left renal vein, since it is only on the left side that adrenal blood drains into the renal vein. In the ideal experiment, the catheter was placed in two positions. In one the tip of the catheter was pushed out as far as it would go with ease. This position was distal to the entrance of the adrenal vein, and was intended as a control on the excretion of the adrenal steroids by the kidney (Figure 2, A). The second position was as close as possible to the junction of the left renal vein and the inferior vena cava. This position was proximal to the usual point of entry of the adrenal vein, and made it possible to sample a mixture of renal and adrenal venous blood (Figure 2, B). Throughout the remainder of the paper, these two positions of the catheter tip will be referred to as RVA and RVB, respectively.

After the catheter had been placed deep in the renal vein, a priming dose of $6.7 \mathrm{mg}$. per $\mathrm{kg}$. of sodium paraamino hippurate (PAH) was given intravenously, after which an infusion of $200 \mathrm{mg}$. of PAH in $100 \mathrm{ml}$. of saline was injected into the vein by an accurately controlled electrical pump at a rate of about one $\mathrm{ml}$. per minute. An indwelling arterial needle was inserted, and a period of 30 to 45 minutes was then permitted to elapse so that the PAH concentration in the blood could become stable, and so that the subject could relax after the relatively minor trauma of the initial manipulations. All instrumentation was performed under adequate metycaine local anesthesia, and none of the patients complained of pain at any time during the procedure.

After the 30-minute equilibration period, one $\mathrm{ml}$. of heparin was injected into the catheter. Samples were

TABLE III

Effect of adrenocorticotrophin (ACTHAR Armour) on the concentration of plasma 17-hydroxycorticosteroids

\begin{tabular}{|c|c|c|c|}
\hline & \multicolumn{2}{|c|}{$\begin{array}{l}\text { 17-hydroxycorticosteroid concentration } \\
\mu g . / 100 \text { ml. plasma }\end{array}$} & \multirow{2}{*}{$\begin{array}{c}\text { Interval } \\
\text { between } \\
\text { injection and } \\
\text { 2nd sample }\end{array}$} \\
\hline & Control & After ACTH & \\
\hline $\begin{array}{l}1 \\
2 \\
3 \\
4\end{array}$ & $\begin{array}{r}5.0 \\
13.0 \\
4.7 \\
8.6\end{array}$ & $\begin{array}{l}17.3 \\
15.7 \\
12.7 \\
10.4\end{array}$ & $\begin{array}{l}4 \text { hrs. } \\
4 \text { hrs. } \\
4 \text { hrs. } \\
10 \text { min. }\end{array}$ \\
\hline
\end{tabular}

Each subject received 25 units of ACTH intravenously immediately after the control sample was drawn.

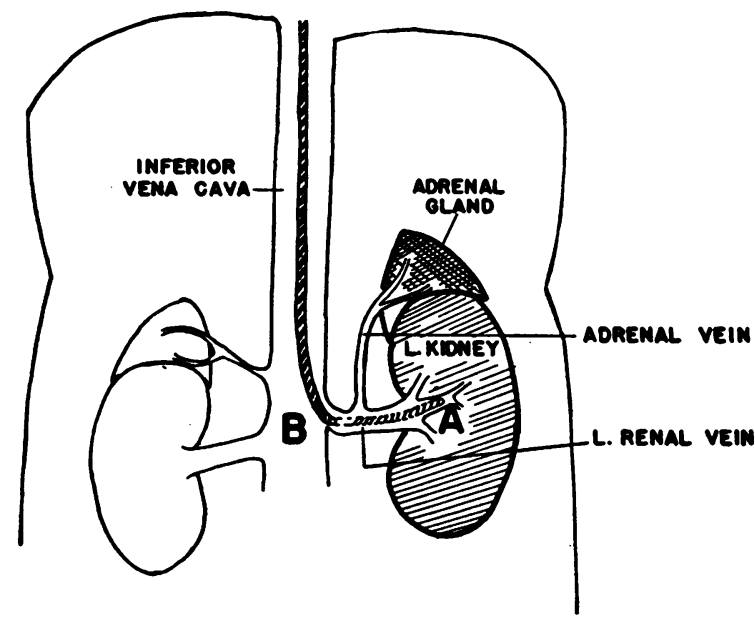

Fig. 2. Positioning of the Catheter

At "A," pure renal venous blood is obtained.

At " $B$," adrenal vein blood is mixed with the venous blood.

taken from the artery and catheter for PAH determination before and after each 17-hydroxycorticosteroid sample. Corticosteroid samples were taken from the catheter and, immediately thereafter, from the artery, first with the catheter tip distal to the entrance of the adrenal vein (Position A, Figure 2), and (usually about five minutes later) with the tip proximal to the adrenal vein (Position B, Figure 2). In some instances, 25 units of ACTH were given intravenously after the second corticosteroid sample, and a third set of samples was taken 5 to 10 minutes later.

Calculations. The renal plasma flow was estimated by the Fick principle, assuming that the only route of exit for PAH from the blood is via the kidneys. This assumption is supported by the fact that the concentration of PAH in samples taken from various veins was indistinguishable from the concentration in the artery. A correction for changing arterial PAH level could have been made by the method suggested for hepatic blood flow estimations by Bradley, Ingelfinger, Bradley, and Curry (9), but arterial PAH levels were sufficiently constant that this correction would have been negligible, and it was therefore ignored. The renal plasma flow was therefore calculated from the formula:

$$
\mathrm{RPF}=\frac{\mathrm{I}}{\mathrm{A}-\mathrm{RV}}
$$

where $R P F=$ renal plasma flow, $I=$ the rate of infusion of PAH in mg. per minute, and $A$ and $R V=$ arterial and renal venous concentrations of PAH in $\mathrm{mg}$. per $\mathrm{ml}$. Since the value for "I" is critical, it was obtained by collecting the outflow from the infusion pump for exactly five minutes at the end of each experiment, analyzing the effluent for PAH, and dividing the result by 5 .

The method of estimating renal plasma flow described above actually measures the rate of flow at the tip of the 
TABLE IV

Estimation of the output of 17-hydroxycorticosteroids by the human adrenal gland

\begin{tabular}{|c|c|c|c|c|c|c|c|c|c|c|}
\hline \multirow[b]{2}{*}{ Name } & \multirow{2}{*}{$\begin{array}{l}\text { Age } \\
\text { Sex }\end{array}$} & \multirow[b]{2}{*}{ Wt., kg. } & \multirow{2}{*}{$\begin{array}{l}\underset{\mathrm{RPF}}{\mathrm{RP}} \\
m l . / m i n .\end{array}$} & \multicolumn{5}{|c|}{$\begin{array}{l}\text { 17.Hydroxycorticosteroid concentration } \\
\mu \mathrm{g} .1100 \mathrm{ml} \text {. plasma }\end{array}$} & \multicolumn{2}{|c|}{ Output } \\
\hline & & & & Art. & $\mathbf{R V} \cdot \mathbf{A}^{*}$ & Art. & RV. B* & RV-B*-Art. & $\mu_{8 .} / \min$. & $m g . / 24 \mathrm{hr}$. \\
\hline H. R. & $\begin{array}{l}13 \\
\mathbf{M}\end{array}$ & 56.8 & 762 & & & 10.8 & 12.8 & 2.0 & 15.2 & 22.0 \\
\hline R. C. & $\begin{array}{l}36 \\
\mathbf{M}\end{array}$ & 68.8 & 933 & & & 11.3 & 13.3 & 2.0 & 18.7 & 26.9 \\
\hline J. C. & $\begin{array}{l}30 \\
\mathbf{M}\end{array}$ & 67.1 & 835 & 6.7 & 6.9 & 6.0 & 6.2 & 0.2 & & \\
\hline J. M. T. & 15 & 65.0 & 865 & 15.6 & 15.6 & 20.0 & 21.4 & 1.4 & 12.1 & 17.4 \\
\hline D. P. & 41 & 59.6 & 707 & & & 8.6 & 11.4 & 2.8 & 19.8 & 28.5 \\
\hline \multicolumn{11}{|c|}{$\begin{array}{c}\text { After ACTH } \\
\text { 17-OH-corticosteroid concentration }\end{array}$} \\
\hline J. M. T. & $\begin{array}{l}15 \\
\mathbf{M}\end{array}$ & 65.0 & 935 & 15.3 & & & 21.3 & 6.0 & 56.1 & 80.8 \\
\hline D. P. & $\begin{array}{l}41 \\
M\end{array}$ & 59.6 & 1257 & 10.4 & & & 12.8 & 2.4 & 30.2 & 43.5 \\
\hline
\end{tabular}

* RV-A and RV-B refer to positions of the catheter shown in Figure 2.

catheter, since dilution of pure renal venous blood by contaminating (e.g., adrenal, ovarian or spermatic vein) blood increases the concentration of PAH in RV. This will decrease the value of $A-R V$, and increase the estimated plasma flow.

The adrenal corticosteroid output was estimated by subtracting the arterial 17-hydroxycorticosteroid concentration from the renal venous concentration, and multiplying the result by the renal plasma flow. The assumptions implicit in this calculation will be discussed below.

\section{RESULTS}

The results obtained in five experiments are shown in Table IV. In each case except one, the concentration of 17-hydroxycorticosteroids was higher in the RV-B sample than in the corresponding arterial sample. Two possible explanations can be suggested for the absence of a difference in J. C. It is possible that the catheter tip was improperly placed, so that sample "RV-B" was actually taken distal to the point of entry of the adrenal vein. It is also possible that this patient had an anomalous adrenal vein which entered the vena cava instead of the left renal vein.

It is noteworthy that the arterial concentration of plasma 17-hydroxycorticosteroids was not elevated above normal levels in four of the five subjects. After the injection of ACTH, the estimated adrenal corticosteroid output was appreciably increased in the two subjects in whom it was possible to perform this experiment. In one case, however
(D. P.), the increase was entirely a result of increased renal plasma flow.

\section{DISCUSSION}

Several fundamental assumptions must be made before the data presented above can be accepted at face value. The most important of these is that adrenal venous blood is uniformly mixed with renal venous blood by the time it reaches the catheter tip. It is highly unlikely that this occurs, since the flow of rapidly moving blood masses tends to stream in discrete layers. The proportions of admixture of the renal and adrenal blood will depend entirely upon the position of the orifice of the catheter tip in relation to the margins of the two streams. Since, however, the renal blood flow is very rapid, and since the presence of the catheter itself probably produces some turbulence, a considerable, unpredictable degree of mixing may occur. To some extent, therefore, the results obtained by the catheter will be determined by chance.

The catheter technique samples a mixture of blood from the left adrenal and kidney. The method of calculation of renal plasma flow assumes that left and right kidneys are equally active and that similar PAH extractions would be obtained if both renal veins were samples. The calculated RPF is, therefore, the total plasma flow for both kidneys. Since the actual observations are made 
only on the left side, it must be assumed that the activity of left and right adrenals is equal. The method of calculation, in fact, results in a value for double the output of the left adrenal, rather than the combined output of right and left adrenal glands. While it is probably correct to assume that the activity of the two glands is usually equal, this is not known in any given case, and grossly incorrect results would be obtained if either gland were very much more active than the other.

The volume of blood flowing through the kidney is so large that the volume of adrenal venous blood added is not significant in disturbing the calculation of renal plasma flow. In most cases, more than 95 per cent of the PAH was removed from the blood obtained through the catheter, indicating that more than 95 per cent of the blood had been exposed to the excretory activity of the renal tubules. This fact results in a very great dilution of the adrenal venous blood. Consequently, the differences in the concentration of 17-hydroxycorticosteroids in arterial and renal vein blood are small. In order to calculate the corticosteroid output, this small difference must be multiplied by the very large renal plasma flow. As a result the importance of analytical inaccuracies in the determination of the adrenal steroids is exaggerated many fold. The calculated output of adrenal steroids must, therefore, be accepted only with grave reservations.

In view of the serious criticisms of the experimental approach which have just been mentioned, it is interesting to find that the results obtained are actually reasonable and consistent with previous observations by other methods. The "resting" output of the human adrenal gland in the four experiments which were successful was equivalent to 17 to $29 \mathrm{mg}$. of Compound $\mathrm{F}$ equivalents per day. These values are not very different from the amounts of cortisone ( 15 to $25 \mathrm{mg}$. per day) found suitable for maintenance therapy in patients with adrenal cortical insufficiency (10). The quantities of steroid secreted five minutes after ACTH approximate the dose of cortisone which has been used for the treatment of patients with Addison's disease subjected to operative trauma (100 mg. per day or so). These results also suggest that the administration of ACTH can result in the secretion of quantities of 17-hydroxycorticosteroids comparable to the therapeutic doses of cortisone used in the treatment of arthritis and other inflammatory diseases.

\section{SUMMARY}

A new method is described for estimating the concentration of 17-hydroxycorticosteroids in plasma. The material measured is of adrenal origin, as evidenced by the fact that it is reduced in patients with Addison's disease, increased after the administration of adrenocorticotrophic hormone, and increased in plasma from the adrenal vein as compared with arterial plasma. The normal concentration range is 3 to $13 \mu \mathrm{gm}$. per 100 ml. plasma. The rate of secretion of 17-hydroxycorticosteroids has been estimated in normal human subjects by collecting simultaneous samples of left renal vein and arterial blood, and determining the difference in concentration of the hormone. The rate of release of the hormones can be estimated by multiplying this difference by the renal plasma flow. In the experiments described, the hormone was secreted at a rate equivalent to 15 to $25 \mathrm{mg}$. of hydrocortisone per day. The limitations of the method are discussed at length. In view of the inherent inaccuracies of the technique, other methods of evaluating the effect of various agents on adrenal secretion must be found.

\section{REFERENCES}

1. Venning, E. H., Kasmin, V. E., and Bell, J. C., Biological assay of adrenal corticoids. Endocrinology, 1946, 38, 79.

2. Paschkis, K. E., Cantarow, A., Walkling, A. A., and Boyle, D., Adrenal cortical hormone levels in adrenal vein-and peripheral blood. Endocrinology, 1950, 47, 338.

3. Porter, C. C., and Silber, R. H., A quantitative color reaction for cortisone and related 17, 21-dihydroxy20-ketosteroids. J. Biol. Chem., 1950, 185, 201.

4. Nelson, D. H., Samuels, L. T., Willardson, D. G., and Tyler, F. H., The levels of 17-hydroxycorticosteroids in peripheral blood of human subjects. J. Clin. Endocrinol., 1951, 11, 1021.

5. Reich, H., Nelson, D. H., and Zaffaroni, A., Isolation of 17-hydroxycorticosterone from blood obtained from adrenal veins of dogs. J. Biol. Chem., 1950, 187, 411.

Bush, I. E., Methods of paper chromatography of steroids applicable to the study of steroids in mammalian blood and tissues. Biochem. J., 1952, 50, 370. 
Savard, K., Kolff, W. J., and Corcoran, A. C., Corticosteroids of peripheral blood. Endocrinology, 1952, 50, 366.

6. Pabst, M. L., Sheppard, R., and Kuizenga, M. H., Comparison of liver-glycogen deposition and work performance tests for the bio-assay of adrenal cortex hormones. Endocrinology, 1947, 41, 55.

7. Dorfman, R. I., Shipley, R. A., Ross, E., Schiller, S., and Horwitt, B. N., The relative potencies of adrenal cortical steroids as determined by a coldprotection test and by a glycogen deposition test. Endocrinology, 1946, 38, 189.
8. Ingle, D. J., Nezamis, J. E., and Morley, E. H., Comparative value of corticosterone, hydrocortisone and adrenal cortex extract given by continuous intravenous injection in sustaining the ability of adrenalectomized rat to work. Am. J. Physiol., 1952, 170, 498.

9. Bradley, S. E., Ingelfinger, F. J., Bradley, G. P., and Curry, J. J., The estimation of hepatic blood flow in man. J. Clin. Invest., 1945, 24, 890.

10. Knowlton, A. I., The modern treatment of Addison's disease. M. Clin. North America, 1952 (May), pp. 721-737. 